\title{
Anatomical vs Functional Preservation of Facial Nerve in a case of Petrous Apex Meningioma
}

\author{
Khan RA ${ }^{1}$, Rahman MM$^{2}$, Khan SIMKN ${ }^{3}$, Kuddus MR $^{4}$,
}

Bang. J Neurosurgery 2021; 10(2): 216-222

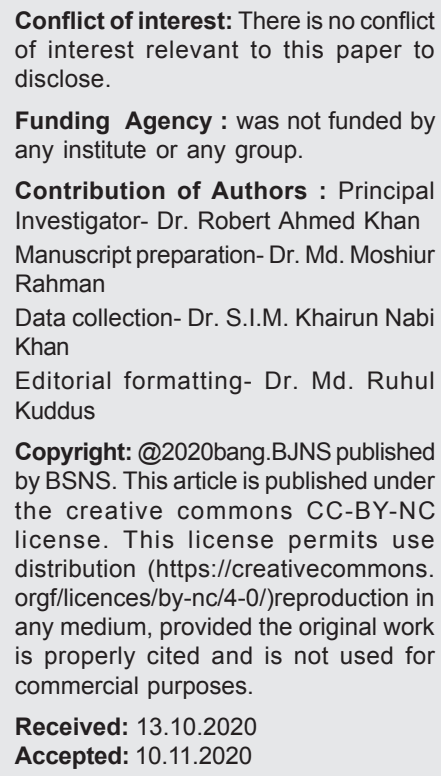

\section{Introduction:}

The first successful removal of cerebellopontine (CP) angle tumor was performed by Sir Charles Balance at the end of $19^{\text {th }}$ century. ${ }^{1}$ On the course time CP angle surgeries have changed from life-saving to function preserving surgeries. Thanks to micro-surgical techniques and intra-operative monitoring (IOM) the preservation of important neural structures especially the facial nerve (FN) has become a rule. But in spite of this advancement good FN function cannot preserved in all CP angle meningiomas especially in premeatal meningiomas ( $76 \%$ preserved in a recent large series). ${ }^{2}$ Petrous apex pathology is generally found by chance on imaging for disconnected side effects. ${ }^{3}$ Symptoms, if present, are heterogeneous.
The most well-known otolaryngologic grievance is hearing loss, yet aural totality and vertigo optional to the pressure of the eighth cranial nerve are additionally normal. ${ }^{4}$ Assessment of petrous apex injuries includes a far-reaching assessment of cranial nerve capacity and audiometry. Computed tomography (CT) is regularly used to decide the ideal careful methodology if careful intercession is justified. Attractive reverberation imaging (MRI) can limit the differential analysis without the requirement for tissue biopsy. ${ }^{5}$ Lesions of the petrous apex are hard to resect because of their profound situation inside the skull base and close anatomical closeness to indispensable structures, for example, the inner carotid supply route (ICA), huge sinus, and Meckel's cavern. Careful choices utilize open, endoscopic, and consolidated strategies arranged into foremost, parallel, and back ways to deal with building up a sufficient passage to the objective thereby. ${ }^{6,7}$

Recent works showed that accomplishing a total tumor resection with the dura and basic bone, relating to Simpson grades I and II, was not measurably associated with a lower pace of repeat in skull base meningiomas. ${ }^{8}$ In a time of multimodal treatment, net all out resection isn't compulsory to accomplish utilitarian conservation and great tumor control. ${ }^{9}$ As careful administration focuses on utilitarian conservation, we chose to focus our series on tumors embedded chiefly on the back substance of the petrous bone and undermining facial and hearing capacities. In this report we discussed a case of petrous apex meningioma where the patient developed immediate post-operative deterioration of FN function in spite of $\mathrm{IOM}$ and anatomical preservation of $\mathrm{FN}$ at the time of

1. Dr. Robert Ahmed Khan, Medical Officer, Neurosurgery Department, BSMMU, Dhaka.

2. Dr. Md. Moshiur Rahman, Assistant Professor, Neurosurgery Department, Holy Family Red Crescent Medical College, Dhaka

3. Dr. S.I.M. Khairun Nabi Khan, Assistant Professor, Neurosurgery Department, BSMMU, Dhaka

4. Dr. Md. Ruhul Kuddus, Medical Officer, Neurosurgery Department, BSMMU, Dhaka

Address of Correspondence: Dr. Md. Moshiur Rahman, Assistant Professor, Neurosurgery Department, Holy Family Red Crescent Medical College, 1, Eskaton Garden Rd, Dhaka 1000, Email: dr.tutul@yahoo.com 
surgery, factors contributing to this morbidity and how it could have been avoided.

\section{Anatomy of the petrous apex}

The petrous apex is a component of the base of the central skull and is classified as the anteromedial portion of the petrous temporal bone to the bony labyrinth and lateral to the petro-occipital fissure. The temporal bone is split into the squamous, petrous, styloid, tympanic, and mastoid sections of the skull base. The most average aspect of the fleeting bone is the petrous segment, which encases the vestibule, crescent channel, facial trench, carotid waterway, and cochlea. It comprises three surfaces and edges, a base and a peak, and is arranged between the greater wing of the sphenoid and the occipital bone. ${ }^{10}$ Within the petrous section, the petrous apex is a triangular region consisting of an anterior apex with borders defined in the upper part by the petrous ridge containing the sulcus of the upper petrosal sinus and in the lower part by the petroclival suture, the lower petrosal sinus groove. A virtual line drawn vertically down from the anterior edge of the porus acousticus is the base of the triangle. ${ }^{11}$ The petrous apex is bounded by the bony labyrinth at the front, the posterior cranial fossa at the back, the Meckel's Cave, and the middle cranial fossa at the top, and the jugular bulb and the lower petrosal sinus at the bottom. ${ }^{12}$ Petrous apex meningioma: petrous apex and adjacent clivus meningiomas are some of the most difficult lesions to treat in the region.

\section{Case report:}

A 36 year old female presented with pain in the left side of the face for the last 2 months. The pain was sharp shooting in nature involving the left half of the upper jaw lasting for a few seconds to a minute. The frequency of attacks increased gradually and recently the pain occurred whenever the patient tried to eat. For this reason the patient became frightful of eating. On query she told of decreased hearing in the left ear with occasional buzzing sound. Clinically she had paresthesia in the left side of the face with no other signs of Vth nerve dysfunction. She had HouseBrackmann ( $\mathrm{HB}$ ) grade II FN palsy with mixed type of deafness on the left side. Her MRI of the brain with contrast showed a contrast enhancing mass in the left $\mathrm{CP}$ angle region with dural base along petrous apex and free margin of tentorial incisura mildly compressing the pons. She underwent left sided suboccipital retrosigmoid craniectomy with $\mathrm{FN}$ monitoring during surgery. After durotomy and drainage of cerebrospinal fluid from the cisterna magna the tumor could be visualized anterior and superior to VII-VIII complex stretching them posteroinferiorly. The FN could be stimulated with a bipolar stimulator at $1 \mathrm{~mA}$ proving the mechanical stretch of the nerve. Later the tumor was removed working over the VII-VIII complex with initial devascularization of the tumor by cauterizing at the base and removal of tumor in piecemeal manner. The total surgery was done under standard free running EMG response and as usual the surgical manipulation was stopped if there was any train response. After

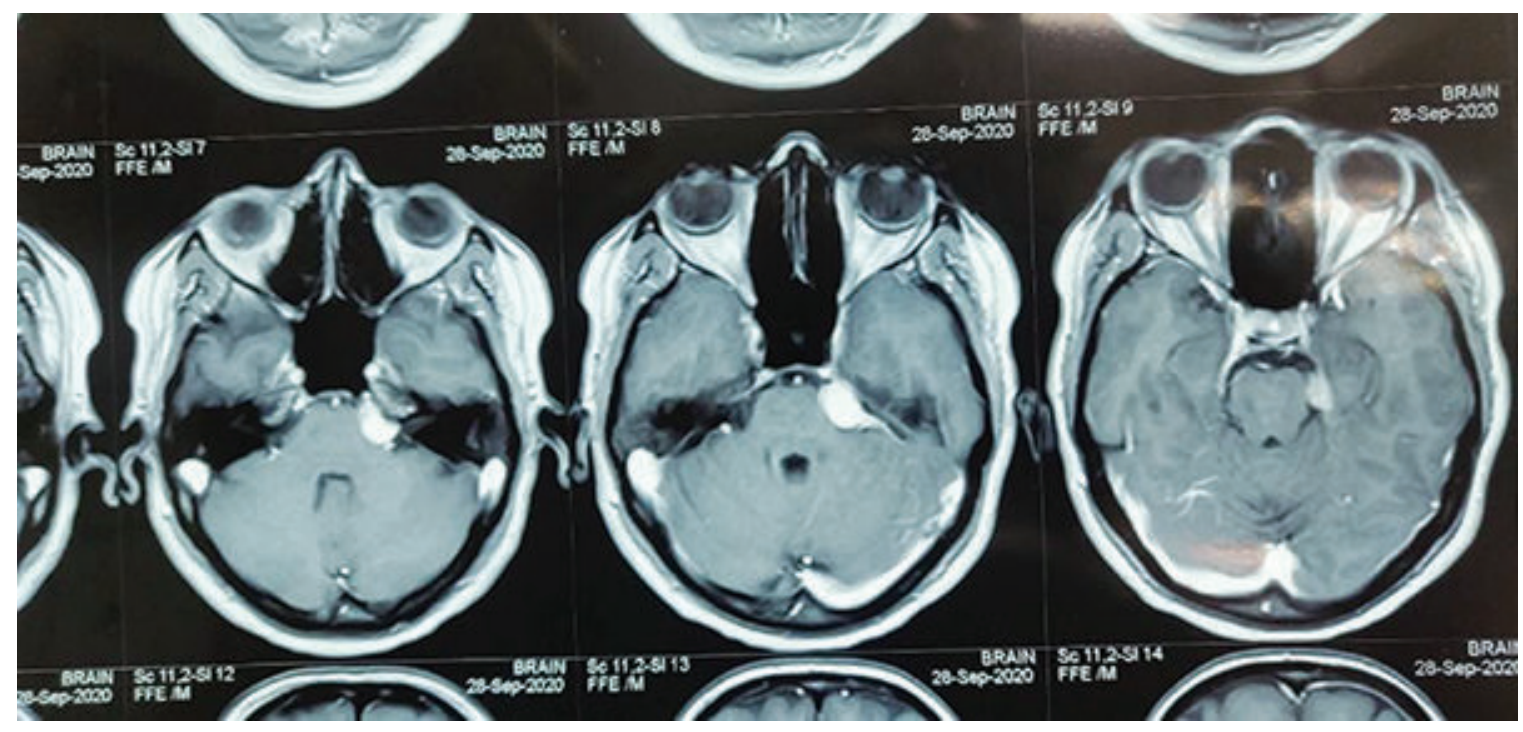

Fig.-1: MRI of Brain with contrast showing contrast lesion left CP angle region. 
removal of the tumor the severely compressed $V$ nerve was visualized with $\mathrm{VI}$ nerve in near vicinity. To determine the functional prognosis of $\mathrm{FN}$ we again stimulated the $\mathrm{FN}$ in its exit zone from the brain stem and its entry into the internal acoustic canal (IAC). There was positive response with same amplitude near the brainstem but poor response near the IAC signifying functional deterioration of $\mathrm{FN}$ function in spite of anatomical preservation at the time of surgery. Immediate post operatively there was complete cessation of the left sided facial pain but the patient developed HB grade III FN palsy. She was discharged with advice of appropriate physiotherapy of the left side of the face with minimum dose of usual post-operative pain killers.

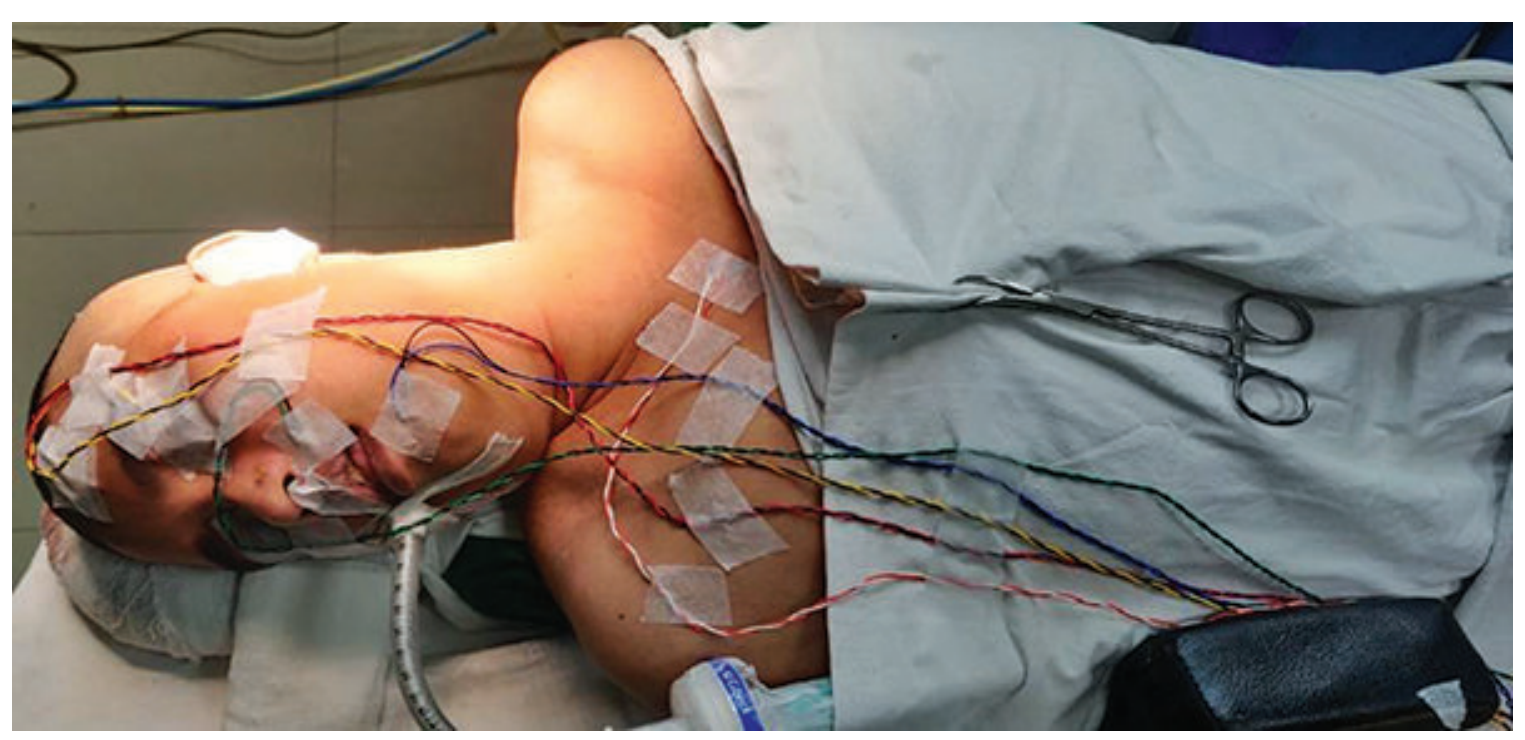

Fig.-2: Patient in operative position with EMG needles in place for facial nerve monitoring.

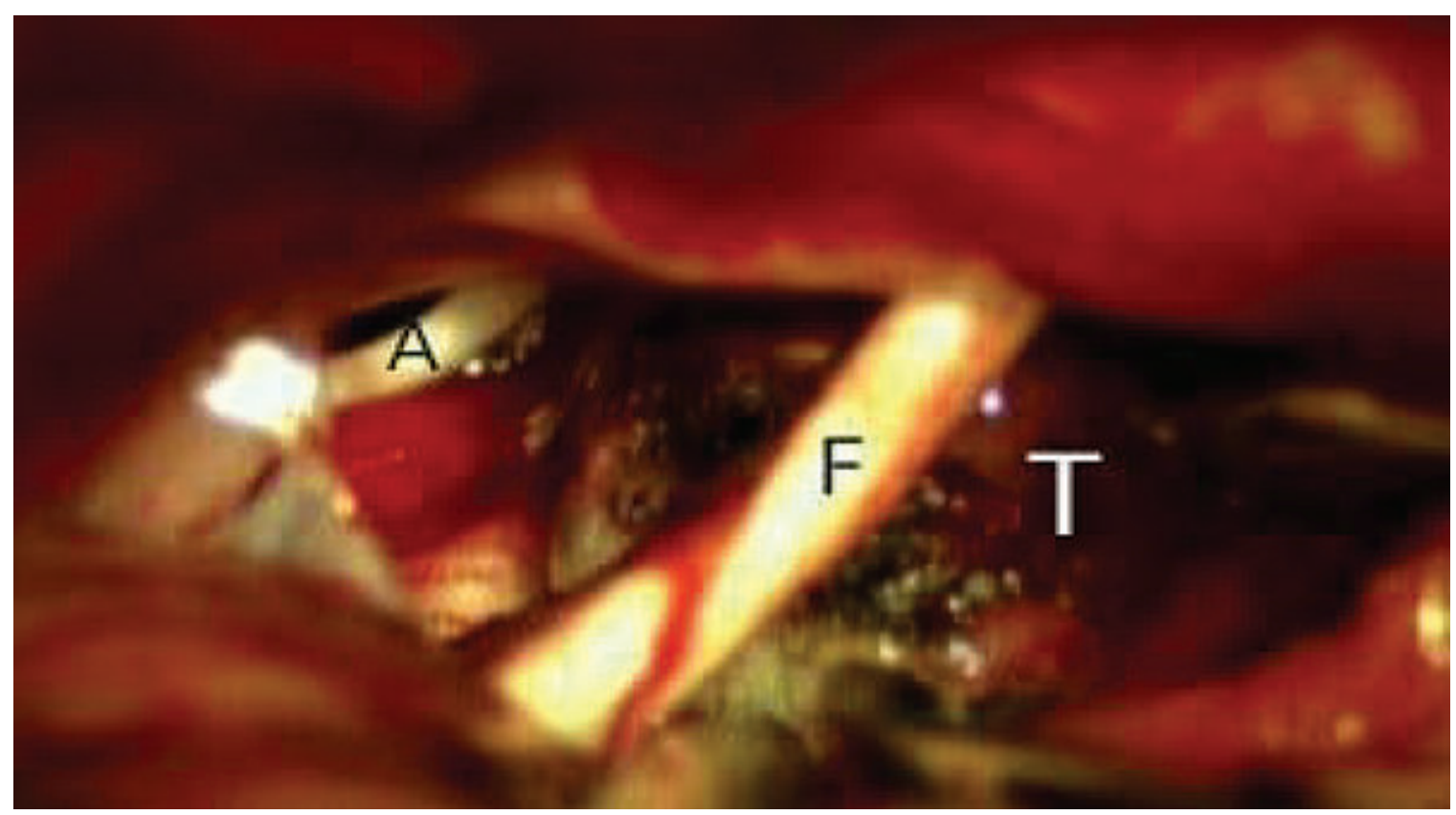

Fig.-3: Operative view of the tumor. $T$ tumor, F facial nerve, $A$ abducens nerve. 


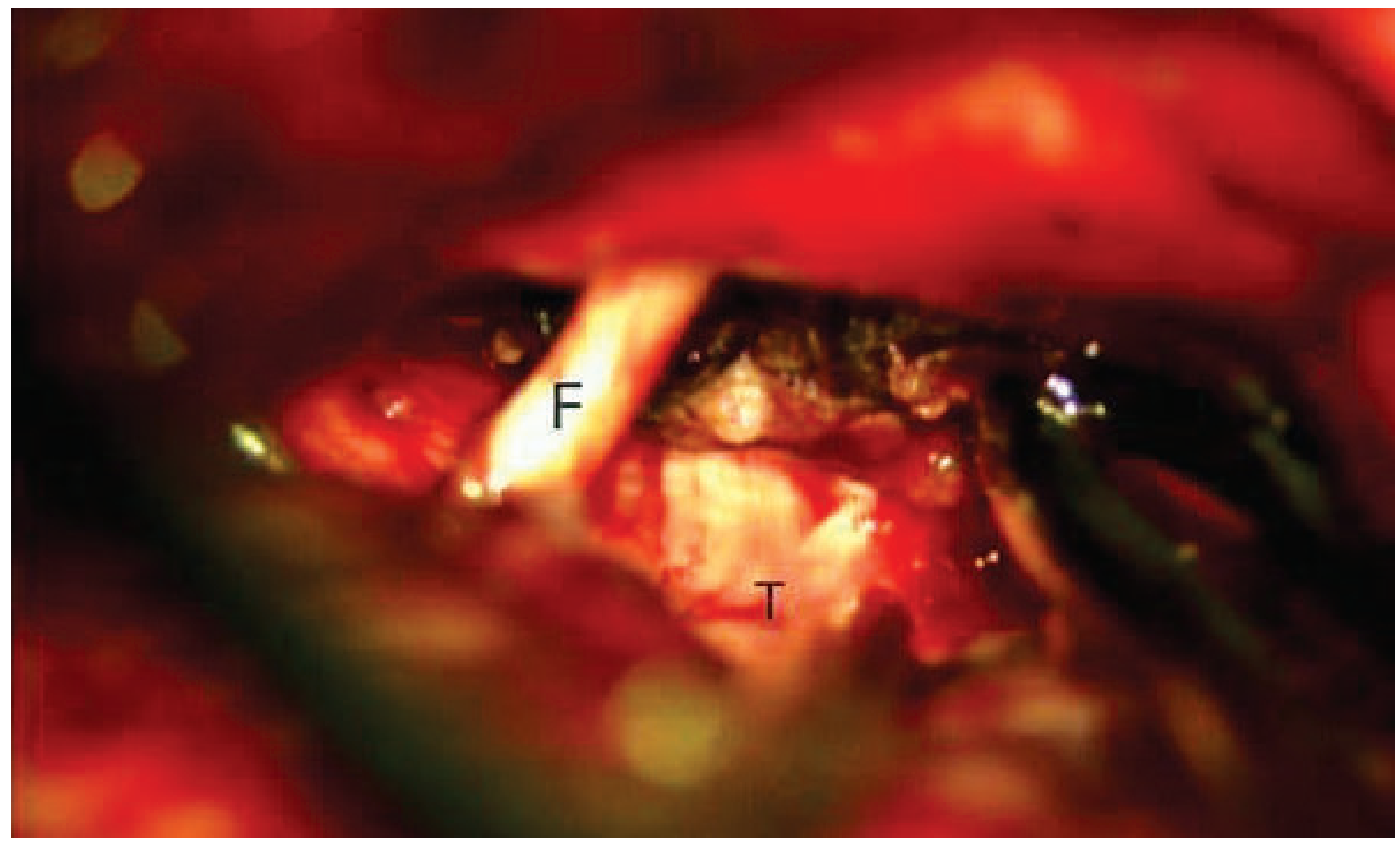

Fig.-4: Trigeminal nerve visualized after removal of tumor, facial nerve remains intact. F facial nerve, $T$ trigeminal nerve.

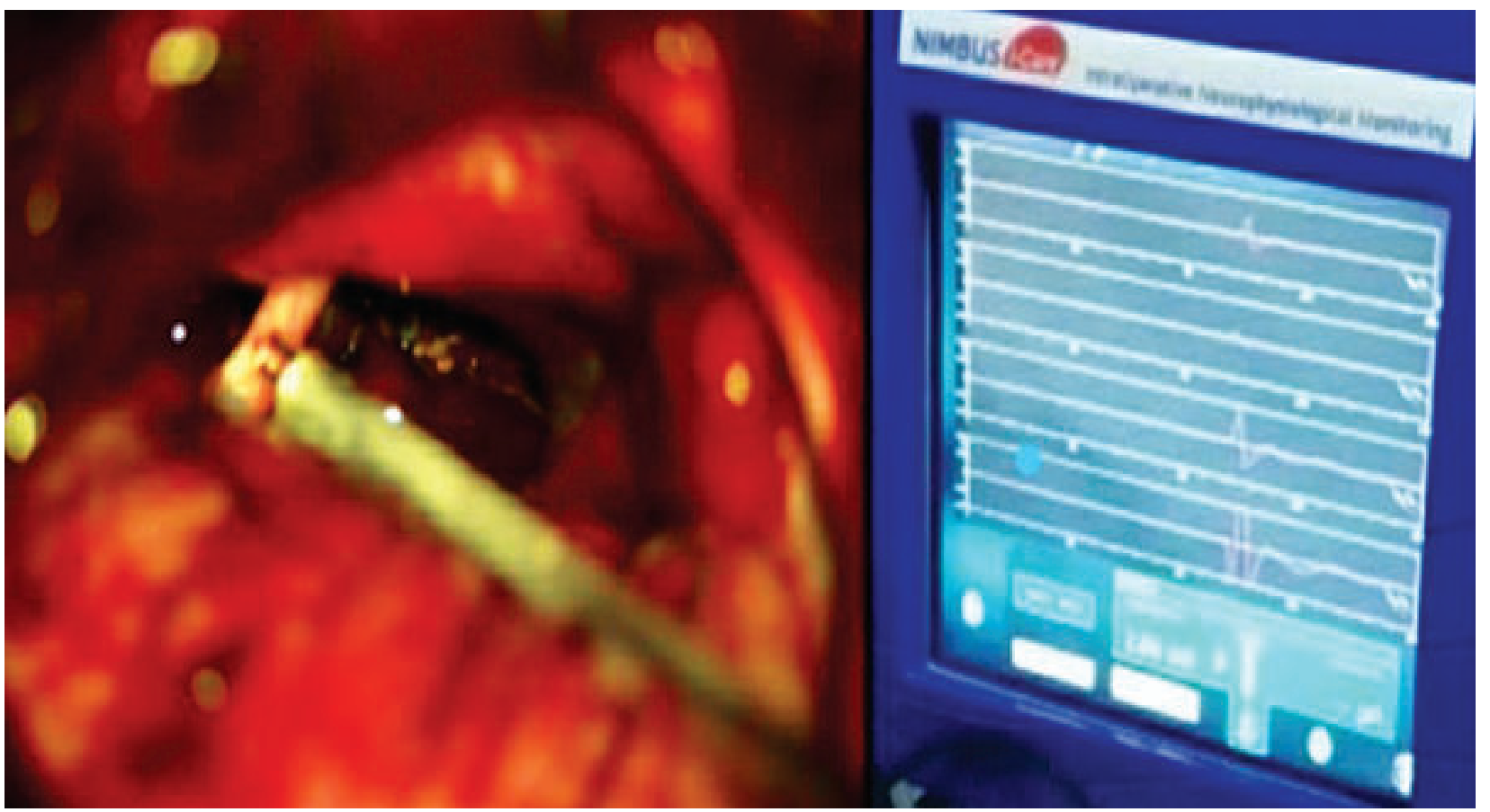

Fig.-5: Direct electrical stimulation of facial nerve. 


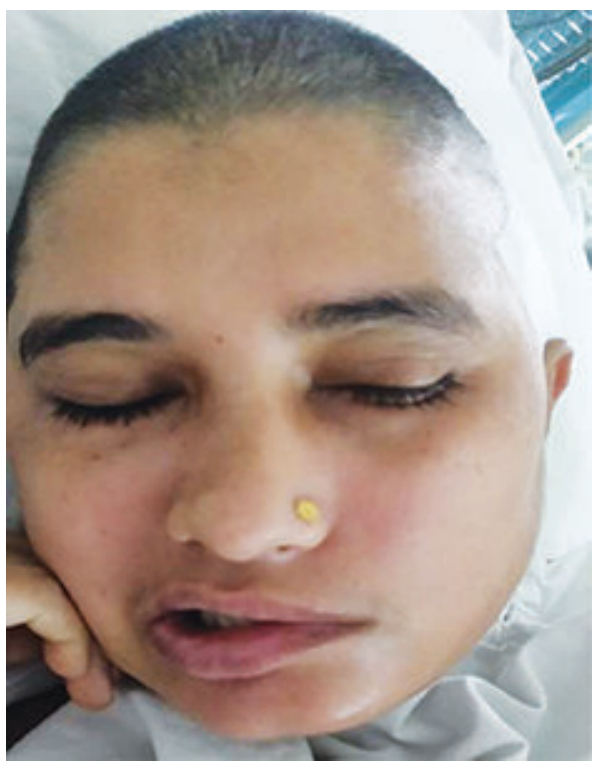

Fig.-6: The patients face immediately after operation.

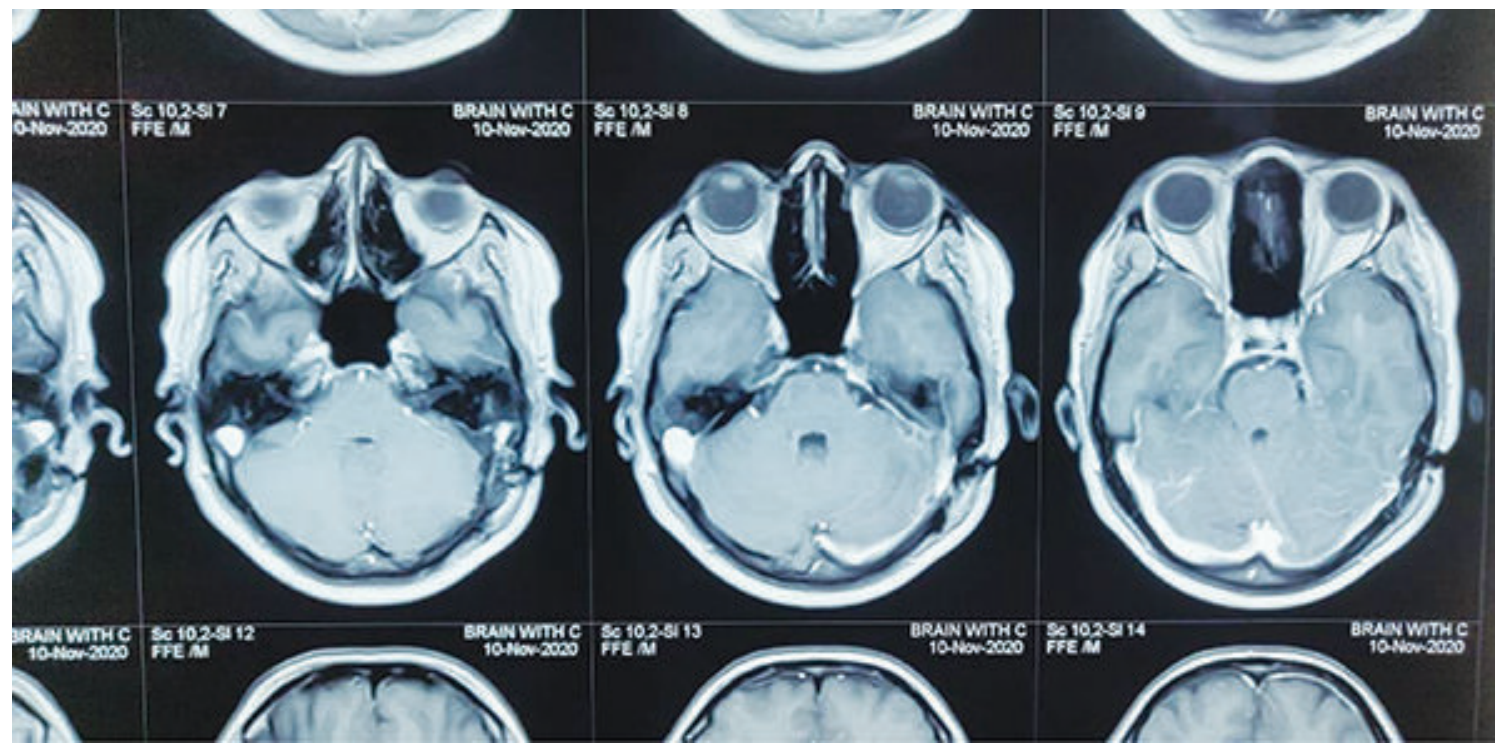

Fig.-7: Postoperative contrast enhanced T1 axial MRI showing no residual.

\section{Head positioning and skin incision}

A three-point pin fixation secures the head and positions it in the lateral position facing away from the surgeon. An incision of periauricular skin in the form of a question mark or $a \mathrm{~L}$ is made. To retract the temporal muscle down and forwards, continue as far as possible in the anterior direction. They are now exposed to the following bony landmarks: mastoid tip, Henle spine, asterion, parietotemporal suture, zygoma base.

\section{Bone drilling and the bone flap}

A retrosigmoid craniectomy of the retromastoid was performed. This superficially exposes, beneath the temporal lobe, the sigmoid sinus and the jugular bulb, the presigmoid dura, and the dura. In-depth, the structure of the semicircular canals can be defined after the removal of the mastoid air cells. When this air cell has been extensively drilled out using a diamond drill, the yellowish compact bone of the lateral semicircular canal is first found at the medial surface 
of the mastoid antrum. For the Fallopian canal, the loop of the lateral semicircular canal is a constant landmark since the second component of the facial nerve passes just below it. To increase the angle of vision, it is important to properly reveal the relief of the posterior labyrinth. A broad temporo-occipital bone flap, exposing the retrosigmoid and temporobasal dura, is then developed. This move enables the dura to be raised from the middle fossa, gradually progressing from the arcuate eminence forward and medially to the Meckel cave. Care should be taken to follow and resect the petrous ridge, continuing forward after raising the dura out of the superior petrosal sinus in the direction of the porus trigeminus.

\section{Retrosigmoid approach}

For tumors that occur at the cerebellopontine angle and include the posterior cranial fossa, the retrosigmoid approach provides excellent access. When tumors reach into the middle fossa and include Meckel's cave, this approach is limited. Cheung and colleagues identified an approach in 1995 that combined retrosigmoid craniotomy with Meckel's cave opening by separating the anterior bone from the internal auditory canal. ${ }^{13} \mathrm{~A}$ series of 12 petroclival meningiomas approached via a similar method, called the retrosigmoid intradural suprameatal method, was later documented by Samii and colleagues. ${ }^{14}$ The suprameatal extension of the retrosigmoid technique allows for better access to the anterior petrous bone of the internal auditory canal and increases vision of the prepontine cistern, clivus, oculomotor nerve, and posterior cerebral arteries. The retrosigmoid approach offers excellent access to tumors arising at the cerebellopontine angle and affecting the posterior cranial fossa. This method is limited if tumors reach into the middle fossa and involve Meckel's cave. In 1995, Cheung and colleagues described a method combining retrosigmoid craniotomy with the opening of Meckel's cave by separating the anterior bone from the inner auditory canal. ${ }^{13}$ Samii and colleagues subsequently reported a series of 12 petroclival meningiomas approached using a similar technique, called the retrosigmoid intradural suprameatal technique. ${ }^{14}$ The suprameatal extension of the retrosigmoid technique allows for greater access to the internal auditory canal anterior petrous bone and improves vision of the prepontine cistern, clivus, oculomotor nerve, and posterior cerebral arteries. ${ }^{15}$

\section{Discussion:}

Although the CP angle was described by Cushing as the "bloody angle", it was Cushing who established new standards of surgery in the posterior fossa and reduced to an acceptable level the complications and the mortality rates of $\mathrm{CP}$ angle surgery.${ }^{16}$ The next major breakthrough in $\mathrm{CP}$ angle tumor surgery was the successful facial nerve preservation. Herbert Olivecrona emphasized the importance of facial nerve preservation. He routinely performed electrical stimulation to identify the nerve and had a special nurse to observe the patient for facial contractions. ${ }^{17}$ Next major breakthrough was the introduction of intraoperative facial nerve monitoring by Delgado et al. in 1979, which opened the way for functional preserving $\mathrm{CP}$ angle surgery. ${ }^{18}$ Since then IOM of FN has become an integral part of $\mathrm{CP}$ angle surgery. Our results suggest that the tumor of the patient requires a more conservative approach for facial and hearing preservation. It is noteworthy that the classification used in this work remains purposely incomplete regarding anatomical locations of meningiomas of the posterior fossa compared with the classification schemes. The locations have been correlated with a better outcome regarding facial function preservation compared with antero-meatal tumors and lesion. ${ }^{19}$ Tumors confined to pure supra- and inframeatal locations are pauci-symptomatic and do not require surgery unless they grow towards the porus and/or become symptomatic. Nowadays, only growing and symptomatic small meningiomas are considered for surgery.

Hearing and facial function preservation is a key feature of the management of pure petrous apex meningiomas as opposed to broad-based petroclival tumors. For pure petrous apex tumors, we advocate the use of an extradural subtemporal approach with apical petrectomy, as those tumors do not invade the Meckel cave. This surgical approach is often considered challenging by surgeons. First, it has been associated with complications due to temporal lobe retraction. ${ }^{20}$ The subtemporal approach is also considered putting the trigeminal nerve at stake while opening the tentorium in clivus meningiomas. Conversely, the use of a retrosigmoid craniectomy approach in petrous apex meningiomas exposes an increased risk of damage to the cochleo-vestibular-facial bundle during surgery, because it represents a continuous obstacle during tumor removal. Injury to the trigeminal nerve in 
this approach may be avoided by rigorous preoperative planning. Some teams defend a systematic use of the retrosigmoid route even in case of preoperative unserviceable hearing, arguing that postoperative recovery may occur. ${ }^{19}$ There is a common agreement that peripetrous approaches should at least be performed whenever a preoperative hearing is serviceable. In this study, the rate of facial nerve function preservation was $100 \%$. These results confirm that among such tumors, large meningiomas are related to worse postoperative facial nerve function. We agree with others that hearing-preserving approaches should always be considered in such approaches but, in our hands, they may not be suitable for large tumors where the facial nerve is more at stake using a retrosigmoid route and gross-total resection is easier to achieve through a transpetrous route. ${ }^{21}$ This reflects the fact that the most frequent tumor remnants are found at the petrous apex and in the Meckel cave.

\section{Conclusion:}

Pathologies of the petrous apex meningioma are a diagnostic and surgical challenge. Surgery is one component in a global multimodal management strategy in which less invasive techniques have their place. In the era of modern skull base surgery, this is no longer any case due to the evolution of excellent surgical approaches aided by advances in neuroradiology, neuroanesthesia, and microsurgery. The retrosigmoid approach for treating such a patient provides favorable outcomes of neurological function. It has the advantage of excellent control of the petrous apex meningioma and a single-stage with preservation of the facial nerve function.

\section{References:}

1. Nguyen-Huynh AT, Jackler RK, Pfister M, Tseng J. The aborted early history of the translabyrinthine approach: a victim of suppression or technical prematurity? Otol Neurotol. 2007 Feb;28(2):269-79.

2. Nakamura M, Roser F, Dormiani M, Matthies C, Vorkapic $P$, Samii M. Facial and cochlear nerve function after surgery of cerebellopontine angle meningiomas. Neurosurgery. 2005 Jul;57(1):77-90.

3. Isaacson B. Cholesterol granuloma and other petrous apex lesions. Otolaryngol Clin North Am. 2015; 48:361e373.

4. Tutar H, Goksu N, Aydil U, et al. An analysis of petrous bone cholesteatomas treated with translabyrinthine transotic petrosectomy. Acta Otolaryngol. 2013; 133:1053e1057.

5. Bruchhage KL, Wollenberg B, Leichtle A. Transsphenoidal and infralabyrinthine approach of the petrous apex cholesterol granuloma. Eur Arch Otorhinolaryngol. 2017; 274:2749e2756

6. Patron V, Humbert M, Micault E, Emery E, Hitier M. How to perform microscopic/endoscopic resection of large petrous apex lesions. Eur Ann Otorhinolaryngol Head Neck Dis. 2018; 135:443e447.

7. Jacquesson T, Berhouma M, Tringali S, Simon E, Jouanneau E. Which routes for petroclival tumors? A comparison between the anterior expanded endoscopic endonasal approach and lateral or posterior routes. World Neurosurg. 2015; 83:929e936.

8. Sughrue ME, Kane AJ, Shangari G, Rutkowski MJ, McDermott MW, Berger MS, Parsa AT (2010) The relevance of Simpson Grade I and II resection in modern neurosurgical treatment of World Health Organization Grade I meningiomas. J Neurosurg 113(5):1029-1035.

9. Ichinose T, Goto T, Ishibashi K, Takami T, Ohata K (2010) The role of radical microsurgical resection in multimodal treatment for skull base meningioma. J Neurosurg 113(5):1072-1078.

10. Rhoton AL. The temporal bone and transtemporal approaches. Neurosurgery. 2000; 47:S211eS265.

11. Fournier HD, Mercier P, Roche PH. Surgical anatomy of the petrous apex and petroclival region. Adv Tech Stand Neurosurg. 2007; 32:91e146.

12. Aubry K, Kania R, Sauvaget E, Huy PT, Herman P. Endoscopic transsphenoidal approach to petrous apex cholesteatoma. Skull Base. 2010; 20:305e308.

13. Cheung SW, Jackler RK, Pitts LH, et al. Interconnecting the posterior and middle cranial fossae for tumors that traverse Meckel's cave. Am J Otol 1995; 16:200-8.

14. Samii M, Tatagiba M, Carvalho GA. Retrosigmoid intradural suprameatal approach to Meckel's cave and the middle fossa: surgical technique and outcome. J Neurosurg 2000; 92:235-41.

15. Chanda A, Nanda A. Retrosigmoid intradural suprameatal approach: advantages and disadvantages from an anatomical perspective. Neurosurgery 2006; 59:ONS1-6 [discussion: ONS1-S6].

16. Cushing $H$ (1917) Tumors of the nervus acusticus and the syndrome of the cerebellopontine angle. WB Saunders Co, Philadelphia.

17. Olivecrona $H$ (1950) Analysis of results of $c$ omplete and partial removal of acoustic neuromas. J Neurol Neurosurg Psychiatry 13(4):271-272.

18. Delgado TE, Bucheit WA, Rosenholtz HR, Chrissian S (1979) Intraoperative monitoring of facila muscle evoked responses obtained by intracranial stimulation of the facila nerve: a more accurate technique for facila nerve dissection. Neurosurgery 4(5):418-421.

19. Nakamura M, Roser F, Dormiani M, Matthies C, Vorkapic P, Samii M (2005) Facial and cochlear nerve function after surgery of cerebellopontine angle meningiomas. Neurosurgery 57(1):77-90, discussion 77-90.

20. . Rosenorn J (1989) The risk of ischaemic brain damage during the use of self-retaining brain retractors. Acta Neurol Scand Suppl 120:1-30.

21. Liu JK, Gottfried ON, Couldwell WT (2003) Surgical management of posterior petrous meningiomas. Neurosurg Focus 14(6):e7. 